\title{
Durability of Cementless Primary Total Hip Arthroplasty in Patients Age 75 and Older
}

\author{
Robert Saldivar ${ }^{1}$, Leonard T. Buller ${ }^{2,3}$, Evan R. Deckard ${ }^{2}$ \\ Mary Ziemba-Davis ${ }^{2}$, R. Michael Meneghini ${ }^{2,3}$ \\ ${ }^{1}$ Indiana University School of Medicine \\ ${ }^{2}$ Indiana University Health Physicians, Orthopaedics \\ ${ }^{3}$ Indiana University School of Medicine, Department of Orthopaedic Surgery \\ Background and Hypothesis: Cementless femoral fixation in total hip \\ arthroplasty (THA) has increased in prevalence worldwide. However, cementless \\ fixation in elderly patients is controversial due to the risk of periprosthetic fracture \\ and/or femoral component loosening. We evaluated the effect of age on implant \\ survivorship in patients $\geq 75$ versus $<75$ years of age. Intraoperative fracture, \\ mortality, and revision cause also were evaluated.
}

Project Methods: 532 cementless THAs performed with consistent surgical, perioperative, and rehabilitation protocols by one surgeon between 2011 and 2018 were retrospectively reviewed. Patients with less than two years follow-up were excluded. 84 patients were $\geq 75$ and 448 were $<75$ years of age. Average follow-up was $44 \pm 12$ months ( $p=0.965)$. Revision rate, intraoperative fracture, 90 day mortality, and overall mortality were compared with $p<0.05$ considered statistically significant.

Results: In the $\geq 75$ group there were more females $(70.2 \%$ vs. $59.2 \%, p=0.067)$, more ASA-PS class 3-4 (76.2\% vs. 46.2\%, p<0.001), and lower BMI $(28.6 \pm 5.7$ vs. $31.3 \pm 6.9, p<0.001)$. Patients $<75$ had more hip dysplasia $(p=0.023)$ and patients $\geq 75$ had more kidney disease $(p<0.001)$. Revision rates between the $\geq 75$ and $<75$ groups $(1.2 \%, 1.8 \%)$ were not different $(p=1.000)$. Moreover, there was no difference in femoral component revision $(<75=62.5 \%, \geq 75=0.0 \%, p=0.444)$ with all femoral revisions due to infection not fracture or loosening. Intraoperative fracture $(<75=0.9 \%, \geq 75=1.2 \%, p=0.578), 90$-day-mortality (one in the younger group, none in the older group, $p=1.000$ ), overall mortality (10 in the younger, 4 in the older group, $p=0.253$ ), and mean months between surgery and death $(p=0.694)$ did not differ in younger and older patients.

Conclusion and Potential Impact: Older patients had comparable implant survivorship compared to younger patients using cementless femoral fixation. In addition, there were no differences in risk for mortality or intraoperative fracture. These findings provide evidence for the safety and durability of cementless THA in elderly patients $\geq 75$ years of age. 